\title{
Training sequence based frequency-domain channel estimation for indoor diffuse wireless optical communications
}

\author{
Jun-Bo Wang ${ }^{1,2^{*}}$, Xiu-Xiu Xie ${ }^{1}$, Yuan Jiao ${ }^{1}$ and Ming Chen ${ }^{3}$
}

\begin{abstract}
Channel estimation is a key technology for wireless optical communication (WOC) systems. Based on the training sequence (TS), this article develops three frequency domain (FD) channel estimation approaches for diffuse wireless optical channels. Considering the propagation property of light and the characteristics of optical modulation, this article establishes a link model for the indoor WOC systems. Using the established link model, three FD channel estimation methods, such as the LS method, the minimum mean square error (MMSE) method and the ORL-MMSE method are proposed. The computational complexity analysis gives time complexities of the three channel estimation methods in FD. This article also evaluates the performance of the proposed three methods by computer simulation, measuring in terms of MSE and BER, respectively. Further more, based on the Cramer-Rao Bound theorem, optimal TSs are found and tabulated for different channel responses and TS lengths. By using the optimal TSs, channel estimation errors measured by BER performance are also investigated.
\end{abstract}

Keywords: Diffuse wireless optical communication (WOC), Frequency-domain (FD) channel estimation, Training sequence (TS), Mean square error (MSE), Cramer-Rao Bound (CRB)

\section{Introduction}

Wireless optical communication (WOC) is an interesting technology combining the radio frequency communications with the traditional infrared communications [1]. Using a light emitting diode (LED) for data transmission, WOC systems can offer several potential advantages over their radio frequency and infrared counterparts, such as security at the physical level, high-data-rate, cost-effective, wide-bandwidth [2]. Therefore, there has been a growing interest in WOC systems in recent years. Despite these advantages, WOC systems for indoor applications are still having many critical issues to be resolved. Generally speaking, influenced by the complicated optical propagation environment, the propagation path between transmitter and receiver is not always unobstructed. Transmit lights were diffusely reflected by reflecting surface, such

\footnotetext{
*Correspondence: jbwang@nuaa.edu.cn

${ }^{1}$ College of Electronic and Information Engineering, Nanjing University of

Aeronautics and Astronautics, Nanjing 210016, China

2 State Key Laboratory of Integrated Services Networks, Xidian University, Xi'an

710071, China

Full list of author information is available at the end of the article
}

as the ceiling, the wall or some other shiny surfaces before they reached to the receiver $[3,4]$. Therefore, the indoor WOC system can be termed as diffusive propagation system. The diffusive WOC system image is drawn in Figure 1. Such diffuse propagation may cause the intersymbol-interference (ISI), which is a primary impediment to communication at high speeds $[5,6]$. In order to eliminate the effects of ISI, the instantaneous channel impulse response must be obtained before the recovery of the transmitted information. Obviously, channel estimation plays a critical role in diffuse WOC systems.

To the best of the authors' investigation, a preamble training sequence (TS) is often used to estimate the channel impulse response in digital communication systems (as seen in Figure 2). For TS-based channel estimation in high speed wireless communications, there mainly are two different ways to estimate the channel impulses, i.e., time-domain (TD) method and frequency-domain (FD) method $[7,8]$. As a typical channel estimation method, TD channel estimation has been discussed extensively for conventional radio communications $[9,10]$. However,

\section{它 Springer}

(c) 2012 Wang et al; licensee Springer. This is an Open Access article distributed under the terms of the Creative Commons Attribution License (http://creativecommons.org/licenses/by/2.0), which permits unrestricted use, distribution, and reproduction in any medium, provided the original work is properly cited. 


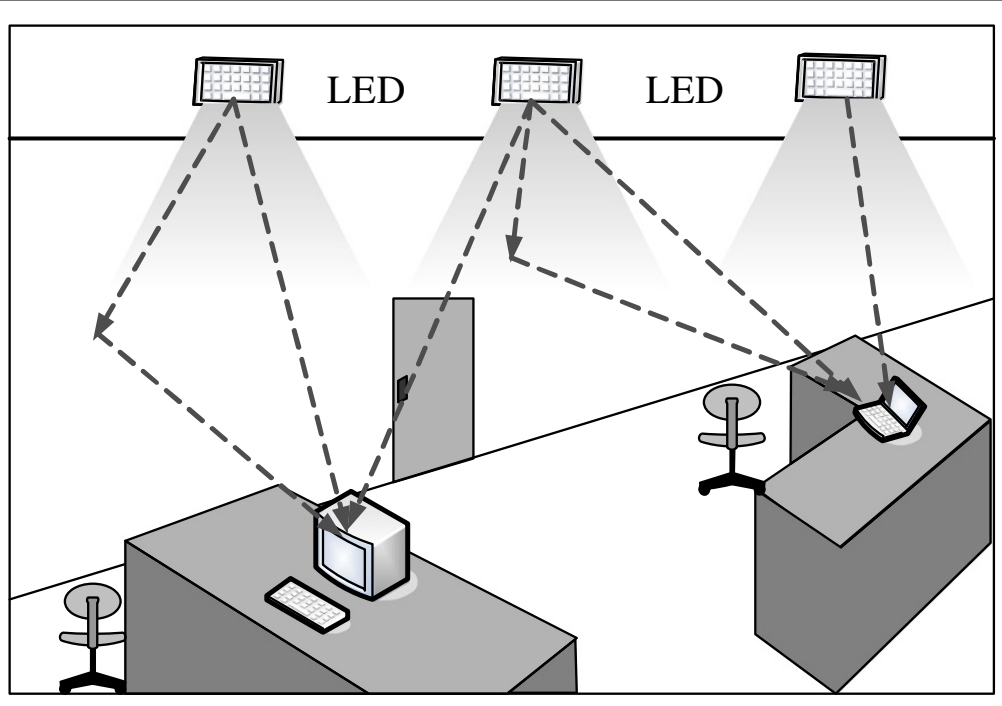

Figure 1 Diffuse WOCs.

only a few works have been done for the channel estimation for diffused WOC systems [11-13]. In [11], TS-based channel estimation for WOC systems has been investigated, and the optimal TSs for a better channel estimation performance have been found by computer search in [12]. Based on the "two-sided" channel estimation, Wang et al. [13] focuses on the complementary sets of sequences for estimating a channel impulse response in WOC systems. Since channel estimation methods in TD are time consuming and the search strategy for optimal TSs based on TD method incurs high computational complexity, a recently proposed channel estimation method based on FD techniques has received enormous attention in radio frequency communications [14,15]. Different with the channel estimation in TD, FD channel estimation denotes the channel impulse response was estimated in the FD, followed by inverse discrete Fourier transform (DFT). To the best of the authors' knowledge, no similar results for channel estimation in WOC systems are available in the open literature.

Motivated by the research [7-15], this article focuses on the TS-based channel estimation methods in FD for WOC systems. Considering the propagation property of light and the characteristics of optical modulation, this article establishes the mathematical channel model for indoor WOC systems. Further, based on the established link model, three FD channel estimation methods, such as the LS method, the minimum mean square error (MMSE) method and the modified MMSE method which can be called optimal low-rank MMSE (OLR-MMSE) are proposed. In addition, this article compares the complexity of the three methods. Furthermore, the performance of the proposed three methods are studied and compared, measuring in terms of Mean square error (MSE) and bit error ratio (BER), respectively. Based on the Cramer-Rao Bound (CRB) theorem, optimal TSs are found and tabulated for different channel responses and TS lengths. By using the optimal TSs, channel estimation errors measured by BER performance are also investigated.

The rest of this article is organized as follows: the mathematical model for WOC systems is described in Section 2. Three channel estimation methods in FD for WOC channels using a know sequence are proposed in Section 3. Section 4 analyzes the computational complexity of the proposed FD channel estimation algorithms. The simulation results of performance of the proposed three channel estimation methods are provided in Section 5. Based on the CRB theorem, Section 6 investigates a valid performance metric for determining optimal TSs. In Section 7,

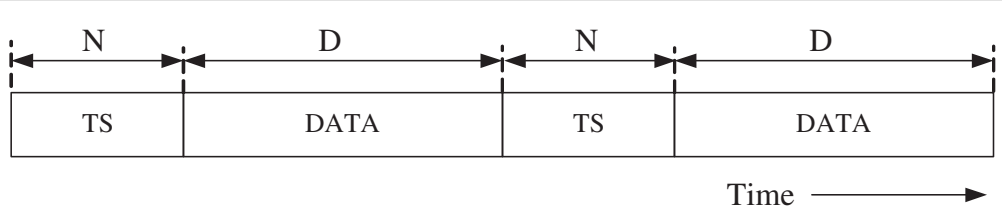

Figure 2 The structure of data frame with TS. 
optimal TSs are obtained by enumerative search and the simulation results of BER performance are shown. Conclusions are drawn in Section 8.

\section{Mathematical model in frequency domain (FD) for diffuse WOCs}

Consider the IM/DD modulation for WOC systems, in which the desired waveform is modulated onto the instantaneous power of the carrier and a photo-detector produces a current proportional to the received instantaneous power. The binary information is modulated into " 0 " and " 1 ". As illustrated in Figure 1, the received signal of the WOC systems is given by [1]

$$
y(t)=a(t) * h(t)+n(t) \quad t \geq 0
$$

where $*$ denotes convolution, $a(t) \in \mathbb{R}^{+} \cup\{0\}$ denotes the instantaneous power in the transmitter, $h(t) \in \mathbb{R}^{+} \cup$ $\{0\}$ is the impulse response of the channel, and $n(t) \in \mathbb{R}$ is the optical channel noise. Digital sampling the continuous signals in the Equation (1), the signal of the $i$ th sampling instant with the sampling period $T_{s}$ can be expressed as

$$
y\left(i T_{\mathrm{s}}\right)=\sum_{m} h\left(m T_{\mathrm{s}}\right) a\left(i T_{\mathrm{s}}-m T_{\mathrm{s}}\right)+n\left(i T_{\mathrm{s}}\right) .
$$

Assuming the channel is represented as a finite impulse response filter with $J$ taps. The channel impulse is given by

$$
h(t)=\sum_{m=0}^{J-1} h_{m} \delta\left(t-m T_{s}\right)
$$

where $\delta(t)$ is the Dirac delta function, $h_{m}$ is $m$ th channel impulse response. Let the initial transmit sequence is $\left\{a_{m} \in\{0,1\} \mid m=0,1, \ldots, N-1\right\}$. Therefore, the received samples described by (2) can be further expressed as

$$
y_{i}=\sum_{m} h_{m} a_{i-m}+n_{i}, \quad i=0,1, \ldots, M-1 .
$$

where $M=N+J-1$ and $\left\{n_{i}\right\}$ denotes the channel noise.

Since, a DFT approach is used to estimate $\left\{h_{m}\right\}$, $\left\{h_{m}\right\}$ and $\left\{a_{m}\right\}$ are augmented such that $h_{m}=0$ for $m=J, J+1, \ldots, M-1$ and $a_{m}=0$ for $m=N, N+$ $1, \ldots, M-1$. The $M$-point DFT of $\left\{a_{m}\right\}$ is defined by the well-known relation

$$
A_{k}=\sum_{m=0}^{M-1} a_{m} e^{-j 2 \pi m k / M}, \quad k=0,1, \ldots, M-1
$$

where $j=\sqrt{-1}$. Similarly, the DFT of $\left\{h_{m}\right\},\left\{y_{m}\right\}$ and $\left\{n_{m}\right\}$ are $\left\{H_{k}\right\},\left\{Y_{k}\right\}$ and $\left\{N_{k}\right\}$, respectively. Assuming the $M$-point DFT of the received signal samples can be denoted as $\mathbf{Y}=\left[Y_{0}, Y_{1}, \ldots, Y_{M-1}\right]^{\mathrm{T}}$. Defining the input matrix $\overline{\mathbf{A}}=\operatorname{diag}\left(\left\{A_{k}\right\}\right)$, the system model in (4) can be described in FD as

$$
\mathbf{Y}=\overline{\mathbf{A}} \mathbf{H}+\mathbf{N} \text {. }
$$

where $\mathbf{H}=\left[H_{0}, H_{1}, \ldots, H_{M-1}\right]^{\mathrm{T}}, \mathbf{N}=\left[N_{0}, N_{1}, \ldots, N_{M-1}\right]^{\mathrm{T}}$ is the Gaussian noise with variance $\sigma^{2}$.

\section{TS based FD channel estimation method}

In data aided channel estimation, the known information to the receiver is inserted in information symbols so that the current channel state can be estimated. By analyzing the relationship between the known TS and the received symbols, the instantaneous channel impulse response can be estimated [16].

\subsection{Least-squares (LS) estimation}

LS channel estimation is commonly used in radio frequency communications when a TS is available. The optimality criterion of this method is to minimize the least square errors to find an optimal estimator to the unknown parameters [9]. It is widely used in practice due to its salient feature that no probabilistic assumptions are made about the parameters. For the channel statistical model in FD as described in (6) for WOC systems, the sum of square error of channel estimation in FD can be defined as

$$
\varepsilon(\mathbf{H})=\|\mathbf{Y}-\overline{\mathbf{A}} \mathbf{H}\|^{2}=(\mathbf{Y}-\overline{\mathbf{A}} \mathbf{H})^{\mathrm{H}}(\mathbf{Y}-\overline{\mathbf{A}} \mathbf{H}),
$$

where $\|\cdot\|$ denotes the norm of a vector, $(\mathbf{x})^{\mathrm{H}}$ is the Hermitian transposition of $\mathbf{x}$. Therefore, the optimality criterion can be defined as

$$
\hat{\mathbf{H}}_{\mathrm{LS}}=\underset{\mathbf{H}}{\arg \min \varepsilon(\mathbf{H})} .
$$

By differentiating with respect to each channel coefficient $\mathbf{H}$ and setting the result to zero, i.e.

$$
\frac{\partial}{\partial \mathbf{H}}(\mathbf{Y}-\overline{\mathbf{A}} \mathbf{H})^{\mathrm{H}}(\mathbf{Y}-\overline{\mathbf{A}} \mathbf{H})=0 .
$$

The closed-form expression for the LS channel estimate in FD can be obtained as

$$
\begin{aligned}
\hat{\mathbf{H}}_{\mathrm{LS}} & =\left(\overline{\mathbf{A}}^{\mathrm{H}} \overline{\mathbf{A}}\right)^{-1} \overline{\mathbf{A}}^{\mathrm{H}} \mathbf{Y}=\overline{\mathbf{A}}^{-1} \mathbf{Y} \\
& =\left[Y_{0} / A_{0}, Y_{1} / A_{1}, \ldots, Y_{M-1} / A_{M-1}\right]^{\mathrm{T}} .
\end{aligned}
$$

Substituting Equation (6) into Equation (10), the LS channel estimate can be expressed as

$$
\hat{\mathbf{H}}_{\mathrm{LS}}=\mathbf{H}+\overline{\mathbf{A}}^{-1} \mathbf{N}
$$

where $\mathbf{H}$ is the objective of the channel estimation, $\overline{\mathbf{A}}^{-1} \mathbf{N}$ denotes the estimated error. It can be observed that, the estimated channel impulse response is free from the interference between taps. In other words, LS channel estimation method is independent of the correlation properties of TS. However, the estimation error variance is largely depending on the channel noise. 


\subsection{MMSE estimation}

Unlike the LS approach, the optimality criterion of MMSE method is to minimize the MSEs to find an optimal estimator to the unknown parameters. Define the linear estimator that achieves the MMSE as

$$
\hat{\mathbf{H}}_{\mathrm{MMSE}}=\mathbf{V Y}
$$

where $\mathbf{V}$ is the linear estimation operator [17]. From the Equation (12), the MSE of the channel estimation for WOC systems can be described as

$\delta(\mathbf{V})=\mathrm{E}\left[\left\|\left(\mathbf{H}-\hat{\mathbf{H}}_{\mathrm{MMSE}}\right)\right\|^{2}\right]=\mathrm{E}\left[\left\|\left(\mathbf{H}-\hat{\mathbf{H}}_{\mathrm{MMSE}}\right)\right\|^{2}\right]$

where $E[\cdot]$ denotes the expectations operator. To obtain the expression of the FD channel estimation, it is expected that the estimation operator $\mathbf{V}$ must be determined at first. Obviously, the optimal operator $\mathbf{V}$ should be satisfying the function as follows

$$
\hat{\mathbf{V}}=\arg \min _{\mathbf{V}} \delta(\mathbf{V}) \text {. }
$$

By differentiating with respect to $\mathrm{V}$ and setting the result to zero, i.e.

$$
\frac{\partial}{\partial \mathbf{V}} \mathrm{E}\left[(\mathbf{H}-\mathbf{V Y})(\mathbf{H}-\mathbf{V Y})^{\mathrm{H}}\right]=0 .
$$

After solving the Equation (15), the close form of expression of operator $\mathbf{V}$ can be written as

$$
\hat{\mathbf{V}}=\mathbf{R}_{\mathrm{H}} \overline{\mathbf{A}}^{\mathrm{H}}\left(\overline{\mathbf{A}} \mathbf{R}_{\mathrm{H}} \overline{\mathbf{A}}^{\mathrm{H}}+\mathbf{R}_{\mathrm{N}}\right)^{-1}
$$

where $\mathbf{R}_{\mathrm{H}}=\mathrm{E}\left[\mathbf{H H}^{\mathrm{H}}\right]$ denotes the autocovariance matrix of channel coefficient in FD, $\mathbf{R}_{\mathrm{N}}=\mathrm{E}\left[\mathbf{N N}^{\mathrm{H}}\right]$ is the covariance matrix of the noise, $(\cdot)^{-1}$ denotes the inverse operation of a matrix. Therefore, the MMSE channel estimate in FD can be expressed as follows

$$
\begin{aligned}
\hat{\mathbf{H}}_{\text {MMSE }} & =\hat{\mathbf{V}} \mathbf{Y}=\mathbf{R}_{\mathrm{H}} \overline{\mathbf{A}}^{\mathrm{H}}\left(\overline{\mathbf{A}} \mathbf{R}_{\mathrm{H}} \overline{\mathbf{A}}^{\mathrm{H}}+\mathbf{R}_{\mathrm{N}}\right)^{-1} \mathbf{Y} \\
& =\mathbf{R}_{\mathrm{H}}\left(\mathbf{R}_{\mathrm{H}}+\sigma^{2}\left(\overline{\mathbf{A}} \overline{\mathbf{A}}^{\mathrm{H}}\right)^{-1}\right)^{-1} \hat{\mathbf{H}}_{\mathrm{LS}} .
\end{aligned}
$$

From the Equation (17), the MMSE approach can alleviate the effect of channel noise in some degree when compared with the LS approach. However, the MMSE channel estimation requires prior knowledge of the channel coefficient covariance matrix $\mathbf{R}_{\mathrm{H}}$ and noise covariance matrix $\mathbf{R}_{\mathrm{H}}$, which means that the MMSE method is more complicated than the LS approach.

\subsection{OLR-MMSE estimation}

The MMSE estimator, as described in (17), is of considerable complexity since a matrix inversion is needed every time the data in $\overline{\mathbf{A}}$ changes. We can reduce the complexity of this estimator by averaging over the transmitted data [15].
The first simplification of MMSE estimator is to replace the term $\left(\overline{\mathbf{A}} \overline{\mathbf{A}}^{\mathrm{H}}\right)^{-1}$ in (17) with its expectation $\mathrm{E}\left\{\left(\overline{\mathbf{A}} \overline{\mathbf{A}}^{\mathrm{H}}\right)^{-1}\right\}$. Assuming the transmitted symbols draw equal-probably. Then, we have

$$
\mathrm{E}\left\{\left(\overline{\mathrm{A}} \overline{\mathrm{A}}^{\mathrm{H}}\right)^{-1}\right\}=\mathrm{E}\left\{\left|1 / A_{k}\right|^{2}\right\} \mathbf{I}
$$

where $\mathbf{I}$ is the identity matrix. Defining the average signal to noise ratio (SNR) as

$$
\overline{\mathrm{SNR}}=\mathrm{E}\left\{\left|A_{k}\right|^{2}\right\} / \sigma^{2} \text {. }
$$

The estimator can be simplified by

$$
\hat{\mathbf{H}}_{\text {MMSE }}=\mathbf{R}_{\mathrm{H}}\left(\mathbf{R}_{\mathrm{H}}+\frac{\beta}{\mathrm{SNR}} \mathbf{I}\right)^{-1} \hat{\mathbf{H}}_{\mathrm{LS}}
$$

where $\beta=\mathrm{E}\left\{\left|A_{k}\right|^{2}\right\} \mathrm{E}\left\{\left|1 / A_{k}\right|^{2}\right\}$. To further reduce the complexity of the estimator, we proceed with the OLR approximations below.

Rank reduction is achieved by using the singular value decomposition (SVD). The SVD of the channel autocovariance matrix is

$$
\mathbf{R}_{\mathrm{H}}=\mathbf{U} \Lambda \mathbf{U}^{\mathrm{H}}
$$

where $\mathbf{U}$ is a unitary matrix containing the singular vectors and $\Lambda$ is a diagonal matrix containing the singular values $\lambda_{1} \geq \lambda_{2} \geq \cdots \geq \lambda_{M}$ on its diagonal. The SVD also dramatically reduces the calculation complexity of matrices.

The system first determines the number of ranks required by the estimator, denoted by $p$, which should be no smaller than $J+1$ [18]. Then, given the noise variance and the channel autocovariance matrix $\mathbf{R}_{\mathrm{H}}$, the receiver pre-calculates $\beta, \mathrm{SNR}$, the unitary matrix $\mathbf{U}$, and the singular values $\lambda_{k}$ s. It thus obtains the $M \times M$ diagonal matrix $\Delta_{p}$ with entries

$$
\delta_{k}=\left\{\begin{array}{l}
{\left[\lambda_{k} /\left(\lambda_{k}+\frac{\beta}{\overline{\mathrm{SNR}}}\right)\right], \quad k=0,1, \ldots, p-1} \\
0=p, \ldots, M-1
\end{array} .\right.
$$

During the transmission, using the transmitted pilot $\overline{\mathbf{A}}$ and $\mathbf{Y}$, the $\hat{\mathbf{H}}_{\mathrm{LS}}$ is calculated according to (10), and the OLR-MMSE estimator with rank $p$ is given by

$$
\hat{\mathbf{H}}_{\mathrm{OLR}-\mathrm{MMSE}}=\mathbf{U} \Delta_{p} \mathbf{U}^{\mathrm{H}} \hat{\mathbf{H}}_{\mathrm{LS}}
$$

The OLR-MMSE estimator can be interpreted as first projecting the LS estimates onto a subspace and then performing the estimation. Because the subspace has a small dimension (as small as $J+1$ ) and still describes the channel well, the complexity of OLR-MMSE estimator is much lower than MMSE estimator with a good performance. 


\section{Computational complexity analysis}

Even when an algorithm is computationally feasible in principle, it may not be realizable in practice if the algorithm requires an inordinate amount of resources (such as running time, storage space, and communication bandwidth) to obtain the solution. Thus, the computational complexity of an algorithm is an important factor for the realization. Moreover, running time is of primary concern in most cases. Generally, the running time needed by an algorithm expressed as a function of the size of the input parameters is called the time complexity of the algorithm [19]. In this section, the computational complexities of the proposed three FD channel estimation algorithms are analyzed in terms of time complexity.

To avoid the effect of running speed, the running time of an algorithm is usually measured by the number of operations needed by the algorithm to obtain the solution. However, in most cases, it is very difficult to determine the exact number of operations of an algorithm. Therefore, using the big- $O$ notation, the time complexity of an algorithm is usually expressed in an asymptotic upper bound on the number of operations [20].

For simplicity, only major computational blocks of the proposed FD estimation algorithms are considered to evaluate the time complexity. Moreover, except for the operations related to the data processing, operations required for matrix formulation, loop counters, assignments of values to variables, etc., are not taken into account. In other words, the time complexity will be measured based on the numbers of multiplication and addition in the major computational blocks. Assuming that the length of TS is $N$, the time complexity is analyzed in Table 1. For a large-enough size of input parameters, the lower order terms of time complexity can be ignored. Therefore, from Table 1, we observe that the LS method is the most time efficient method among the FD algorithms. MMSE algorithm with a highest complexity and the OLRMMSE is efficient than MMSE by reducing the rank of the channel correlation matrix.

\section{Quality measure of FD channel estimate}

To evaluate the performance of the proposed FD channel estimation schemes, the proposed three methods are studied and compared by computer simulation. Here, two comparisons are considered. Firstly, the proposed three methods are compared in terms of MSE, which denotes

Table 1 Complexity comparison

\begin{tabular}{lcc}
\hline Channel estimation method & Multiplication & Addition \\
\hline FD-LS & $O(M)$ & $O(1)$ \\
FD-MMSE & $O\left(M^{3}\right)$ & $O\left(M^{3}\right)$ \\
FD-OLR-MMSE & $O\left(r M^{2}\right)$ & $O\left(r M^{2}\right)$ \\
\hline
\end{tabular}

the accuracy of the channel estimation. Secondly, the three methods are compared, measuring by BER, which denotes the system performance influenced by channel estimation.

In the first comparison, one way of quantifying channel estimation performance is by MSE matrix [9], also referred to as the error covariance. The MSE matrix can be defined as

$$
\mathbf{M S E}=\mathrm{E}\left\{(\mathbf{h}-\hat{\mathbf{h}})(\mathbf{h}-\hat{\mathbf{h}})^{\mathrm{H}}\right\}
$$

where $\mathbf{h}$ and $\hat{\mathbf{h}}$ are the IDFT of $\mathbf{H}$ and $\hat{\mathbf{H}}$, respectively. Without lose of generality, we assume that the length of TS used in channel estimation $N=26$ and the number of channel $\operatorname{taps} J=3$.

Figure 3 depicts the MSE performance versus the SNR with different FD channel estimation methods. It can be seen from Figure 3 that the MSE performance is decreases with the increase of the SNR. In addition, the best and worst MSE performances are achieved by the proposed MMSE channel estimation and the LS estimation algorithm, respectively. Also, for OLR-MMSE estimator, a degraded performance is shown due to the loss of channel information by reducing the rank of the channel correlation matrix. Moreover, since the LS method is sensitive to the signal noise power, the required SNR is $5 \mathrm{~dB}$ higher than the OLR-MMSE method with the MSE constraint and is set to be $10^{-2}$.

Figure 4 shows the MSE performance versus SNR with different estimation errors. With the LS channel estimation method, the MSE performances are free from the effects caused by the coefficient covariance matrix $\mathbf{R}_{\mathrm{H}}$. However, the MSE increases significantly with the existence of the estimation error of $\mathbf{R}_{\mathrm{H}}$ when used MMSE channel estimation method. With the increase of the error variance of $\mathbf{R}_{\mathrm{H}}$, the performance getting worse. Even though the SNR is high, the MSE performance for MMSE estimation is worse than LS estimation when there exits the estimation error for $\mathbf{R}_{\mathrm{H}}$.

Figure 5 shows the second comparison. In Figure 5, the BER performance versus the SNR is depicted for the proposed three FD channel estimation schemes over the diffuse channel of WOC systems. It can be seen from Figure 5 that the BER performance is decreases with the increase of the SNR. The MMSE estimation method yields the best performance, and LS yields the worst. Also, for the OLR-MMSE estimator, a BER floor is shown due to loss of channel information by reducing the rank of the channel correlation matrix.

\section{TS optimization}

The CRB provides a lower bound on the statistical variance of any unbiased estimator, which means that the lower bound on CRB is the minimum variance for an 


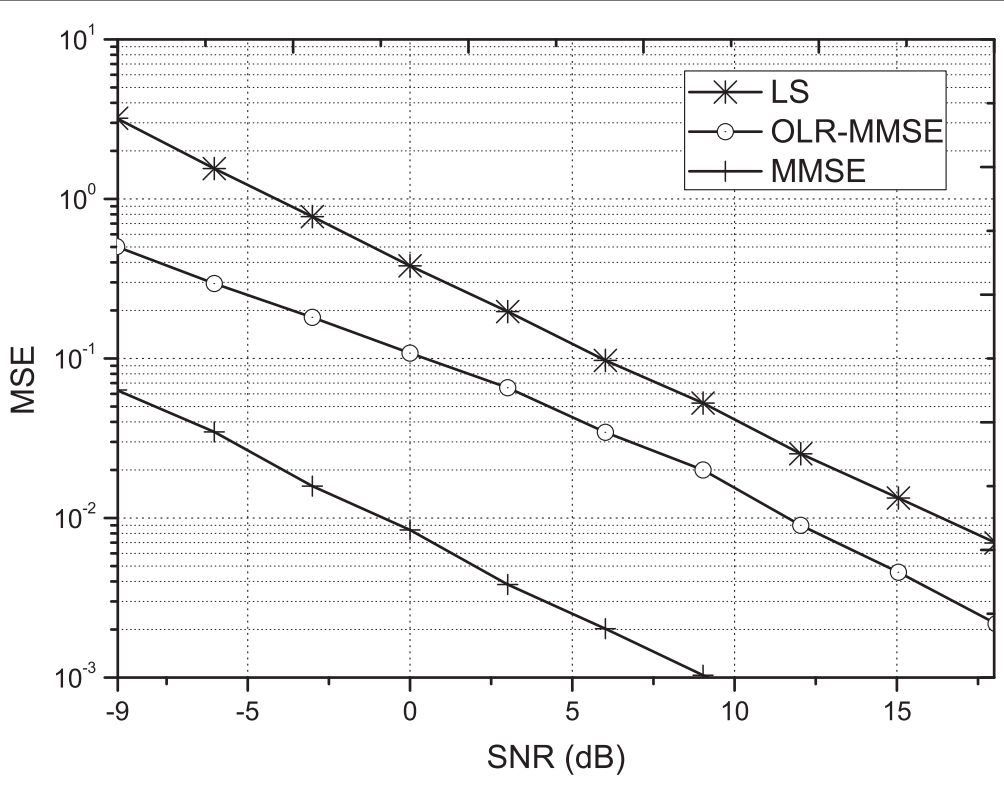

Figure 3 MSE performance versus the SNR with different FD channel estimation methods.

estimator can obtain. The CRB for random channels has been wildly used as a performance measure for the design of optimal TSs $[17,18]$. In this section, we first derive a closed-form expression for the CRB for the estimation of $\mathbf{H}$.

Based on the CRB theorem, the Fisher information matrix (FIM) I (H) is given as follows [21]

$$
\mathbf{I}(\mathbf{H})=\mathrm{E}\left\{\frac{\partial \ln p(\mathbf{Y}, \mathbf{H})}{\partial \mathbf{H}}\left(\frac{\partial \ln p(\mathbf{Y}, \mathbf{H})}{\partial \mathbf{H}}\right)^{\mathrm{H}}\right\}
$$

where $\mathbf{x}^{\mathrm{H}}$ is the Hermitian transposition of $\mathbf{x}, p(\mathbf{Y}, \mathbf{H})$ is the joint probability density function of received signal sequences and channel coefficients, the derivatives are evaluated at the true value of $\mathbf{H}$ and the expectation is taken with respect to $p(\mathbf{Y}, \mathbf{H})$.

As described in the CRB theorem, it is sometimes possible to determine the minimum variance unbiased (MVU) estimator if the equality constraints of the CRB theorem are satisfied. $\hat{\mathbf{H}}=\mathbf{g}(\mathbf{Y})$ will be the MVU estimator if

$$
\frac{\partial \ln p(\mathbf{Y}, \mathbf{H})}{\partial \mathbf{H}}=\mathbf{I}(\mathbf{H})(\mathbf{g}(\mathbf{Y})-\mathbf{H})
$$

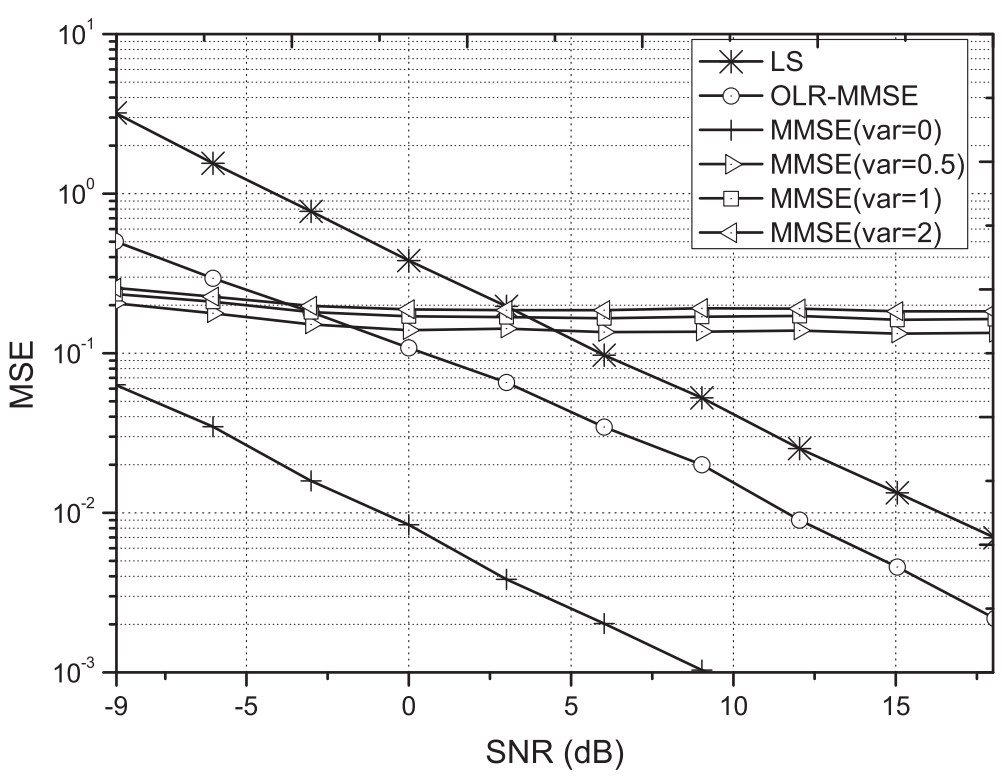

Figure 4 MSE performance versus SNR with different estimation errors. 


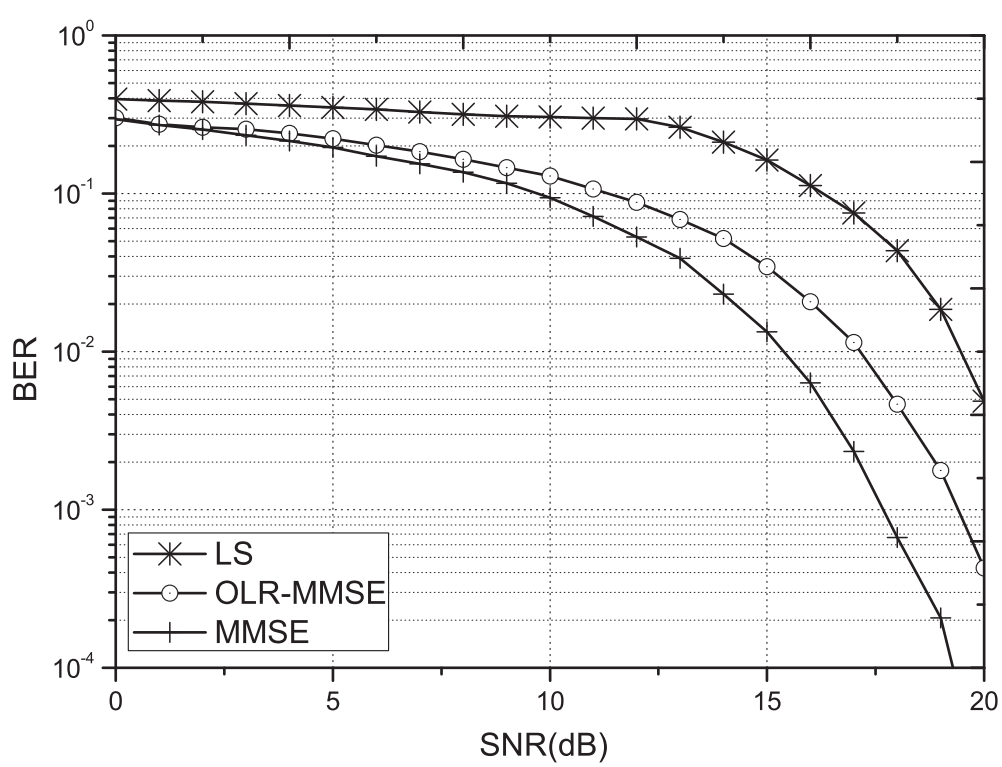

Figure 5 BER performance versus the SNR with different FD channel estimation methods.

for some function $\mathbf{g}$. Furthermore, the covariance matrix of $\hat{\mathbf{H}}$ will be $\mathbf{I}^{-1}(\mathbf{H})$. For the linear channel model of (6), we have

$p(\mathbf{Y}, \mathbf{H})=\frac{1}{\left(2 \pi \sigma^{2}\right)^{L / 2}} \exp \left(-\frac{1}{2 \sigma^{2}} \sum_{n=0}^{M-1}(\mathbf{Y}[n]-\overline{\mathbf{A}} \mathbf{H}[n])^{2}\right)$

then the left part of the Equation (27) can be derived as

$$
\begin{aligned}
\frac{\partial \ln p(\mathbf{Y}, \mathbf{H})}{\partial \mathbf{H}} & =\frac{\partial}{\partial \mathbf{H}}\left[-\ln \left(2 \pi \sigma^{2}\right)^{\frac{L}{2}}-\frac{1}{2 \sigma^{2}}(\mathbf{Y}-\overline{\mathbf{A}} \mathbf{H})^{\mathrm{H}}(\mathbf{Y}-\overline{\mathbf{A}} \mathbf{H})\right] \\
& =\frac{1}{2 \sigma^{2}} \frac{\partial}{\partial \mathbf{H}}\left[\mathbf{Y}^{\mathrm{H}} \mathbf{Y}-2 \mathbf{Y}^{\mathrm{H}} \overline{\mathbf{A}} \mathbf{H}+\mathbf{H}^{\mathrm{H}} \overline{\mathbf{A}}^{\mathrm{H}} \overline{\mathbf{A}} \mathbf{H}\right]
\end{aligned}
$$

where $\sigma^{2}$ is the variance of the Gaussian noise. Using the identities

$$
\begin{aligned}
& \frac{\partial \mathbf{b}^{\mathrm{H}} \mathbf{H}}{\partial \mathbf{H}}=\mathbf{b} \\
& \frac{\partial \mathbf{b}^{\mathrm{H}} \mathbf{S H}}{\partial \mathbf{H}}=2 \mathbf{S} \mathbf{b} .
\end{aligned}
$$

Table 2 Complexity comparison

\begin{tabular}{lcc}
\hline Optimal TS search method & Multiplication & Addition \\
\hline FD method & $O\left(M \log _{2} M\right)$ & $O\left(M \log _{2} M\right)$ \\
TD method & $O\left((M+1) \mathcal{S}^{2}\right)$ & $O\left((M+1) \mathcal{I}^{2}\right)$ \\
\hline
\end{tabular}

The simpler expression of Equation (28) can be expressed as

$$
\frac{\partial \ln p(\mathbf{Y}, \mathbf{H})}{\partial \mathbf{H}}=\frac{1}{\sigma^{2}}\left[\overline{\mathbf{A}}^{\mathrm{H}} \mathbf{Y}-\overline{\mathbf{A}}^{\mathrm{H}} \overline{\mathbf{A}} \mathbf{H}\right] .
$$

Since, $\overline{\mathbf{A}}^{\mathrm{H}} \overline{\mathbf{A}}$ is invertible, (30) can be rewritten as

$$
\frac{\partial \ln p(\mathbf{Y}, \mathbf{H})}{\partial \mathbf{H}}=\frac{\overline{\mathbf{A}}^{\mathrm{H}} \overline{\mathbf{A}}}{\sigma^{2}}\left[\left(\overline{\mathbf{A}}^{\mathrm{H}} \overline{\mathbf{A}}\right)^{-1} \overline{\mathbf{A}}^{\mathrm{H}} \mathbf{Y}-\mathbf{H}\right]
$$

which is exactly in the form of (26) with

$$
\hat{\mathbf{H}}=g(\mathbf{Y})=\left(\overline{\mathbf{A}}^{\mathrm{H}} \overline{\mathbf{A}}\right)^{-1} \overline{\mathbf{A}}^{\mathrm{H}} \mathbf{Y}
$$

$$
\mathbf{I}(\mathbf{H})=\frac{\overline{\mathbf{A}}^{\mathrm{H}} \overline{\mathbf{A}}}{\sigma^{2}} .
$$

Hence, the MVU estimator of $\mathbf{H}$ is given by (32a), and its covariance matrix is

$$
\mathbf{C}_{\hat{\mathrm{H}}}=\mathbf{I}^{-1}(\mathrm{H})=\sigma^{2}\left(\overline{\mathbf{A}}^{\mathrm{H}} \overline{\mathbf{A}}\right)^{-1} .
$$

Further, the CRB of channel estimation in FD is given by

$$
\begin{aligned}
\mathrm{CRB}_{\mathrm{F}-\mathrm{OP}} & =\operatorname{tr}\left(\sigma^{2}\left(\overline{\mathbf{A}}^{\mathrm{H}} \overline{\mathbf{A}}\right)^{-1}\right)=\sigma^{2} \operatorname{tr}\left(\left(\overline{\mathbf{A}}^{\mathrm{H}} \overline{\mathbf{A}}\right)^{-1}\right) \\
& =\sigma^{2} \sum_{k=0}^{M-1} \frac{1}{\left|A_{k}\right|^{2}}
\end{aligned}
$$


Table 3 Hexadecimal value of optimal TS

\begin{tabular}{|c|c|c|c|c|c|}
\hline \multirow{2}{*}{$\begin{array}{l}\text { Channel } \\
\text { order J }\end{array}$} & \multicolumn{5}{|c|}{ TS length $(N)$} \\
\hline & $2 J$ & $2 J+1$ & $2 J+2$ & $2 J+3$ & $2 J+4$ \\
\hline$J=2$ & $\mathrm{~F}$ & 13 & 27 & $3 D$ & $\mathrm{BC}$ \\
\hline$J=3$ & $3 D$ & $5 \mathrm{E}$ & 97 & OB7 & $35 \mathrm{~F}$ \\
\hline$J=4$ & ED & 08B & $20 B$ & $3 \mathrm{DD}$ & F59 \\
\hline$J=5$ & $3 \mathrm{DD}$ & 765 & DAB & 1677 & $2 \mathrm{~A} 6 \mathrm{~F}$ \\
\hline$J=6$ & EB7 & OF35 & $36 \mathrm{A3}$ & $6 \mathrm{D} 77$ & B3AF \\
\hline$J=7$ & $32 \mathrm{~A} 6$ & $2 \mathrm{~B} 67$ & F36A & $15 B 67$ & 23597 \\
\hline
\end{tabular}

where $\operatorname{tr}(\Phi)$ means the sum of the main diagonal elements of matrix $\Phi$. Obviously, (34) can be used to evalute the performance of different TSs. Therefore, the optimal TS can be obtained as

$$
\mathrm{TS}_{\mathrm{F}-\mathrm{OP}}=\arg \min _{\mathbf{a} \in\{0,1\}^{N}}\left(\sigma^{2} \sum_{k=0}^{M-1} \frac{1}{\left|A_{k}\right|^{2}}\right) .
$$

Based on (35) optimal binary sequences are found by exhaustive computer search. The resulting sequences offer the best performance of error covariance at the output of the channel estimator.

Table 2 compares the time complexity of the search methods in FD and TD, respectively. For simplicity, only major computational block of the proposed FD search strategy and TD search strategy are considered to evaluate the time complexity. Comparing the search strategies described in [12] for TD channel estimation method with the FD method described in (35), we can see from the table that the optimal TSs search strategy in FD can significantly reduces the complexity of the search. In other words, the procedure of searching for optimum sequences in FD is time consuming than that of TD method.

\section{Enumerative computer search for optimal TSs}

As the placement of $N$ clusters of pilot symbols is shown in Figure 2, a computer search has been performed to find the FD optimal TSs satisfying the Equation (35) for a given number of channel order $J$ and TS length $N$.

Table 3 lists the optimal sequences found up to length 18. The sequences are binary and are represented in hexadecimal form for compactness. The most significant bit corresponds to the first symbol of the TS to be transmitted. The length of the preamble is uniquely determined by its location in the table. If the sequence length is not a multiple of four, as many zeros as is necessary are added or neglected to obtain the correct preamble length.

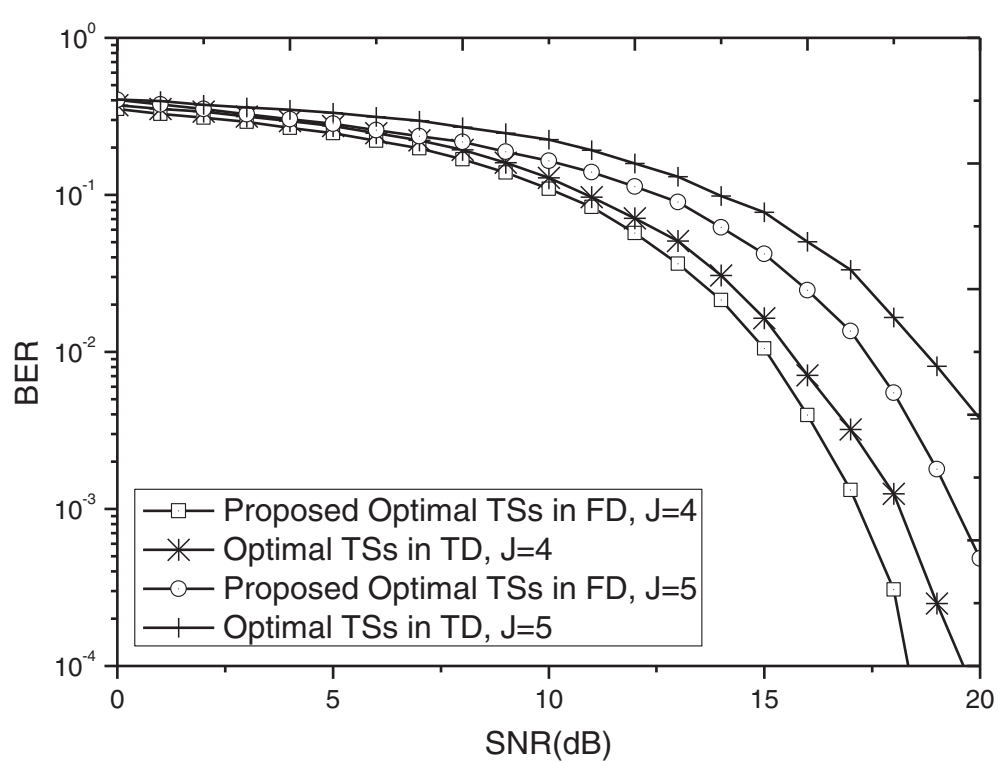

Figure 6 BER performance versus the SNR by using proposed optimal TS and optimal TS in TD, respectively. $N=12$ 


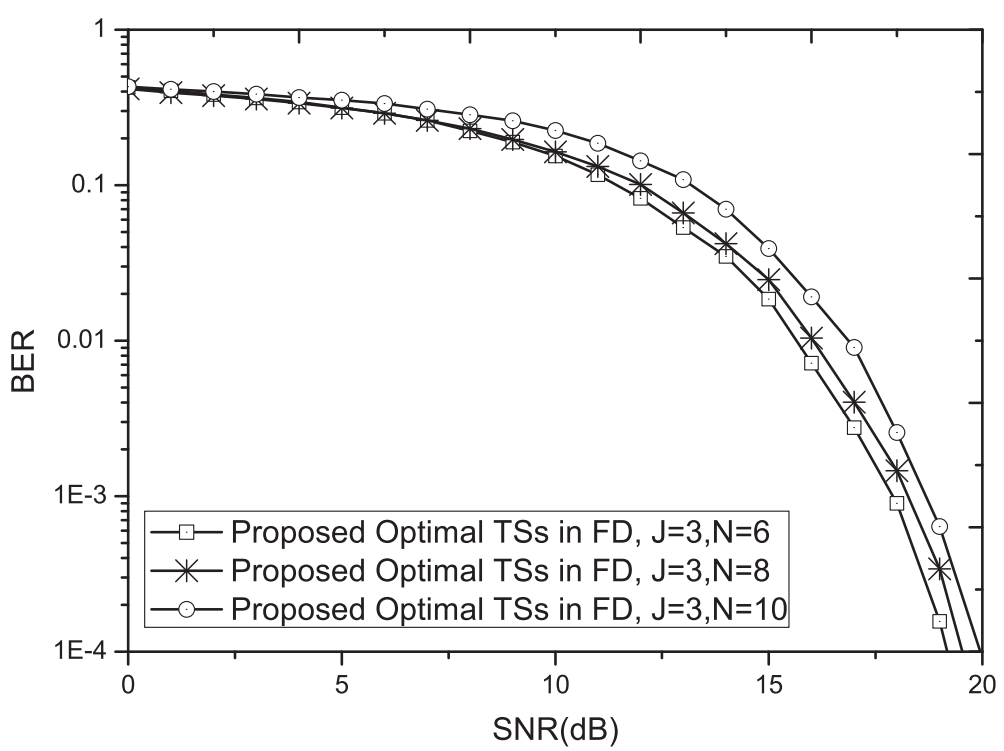

Figure 7 BER performance versus the SNR by using proposed optimal TS in FD. Channel state $J=3$.

To evaluate the performance, it is desired to compare our proposed optimal TSs in FD with the optimal TSs in TD for FD-LS channel estimation in terms of the BER via computer simulations, respectively. Although no TSs in TD have been proposed in published articles for WOC system, in our former research, we have investigated the optimal TSs for TD channel estimation for WOC systems [14].

Figure 6 compares the BER versus the SNR for the FD-LS channel estimation methods with proposed optimal TS and optimal TS in TD, respectively. The number of channel taps are $J=4$ and $J=5$. The TS length is $N=12$. It can be seen from Figure 6 that the BER decreases with the increase of the SNR. With the same length of channel taps, the proposed FD optimal TS with the FD channel estimation can obtained a better BER performance compared with the optimal TS in TD method. In other words, the optimal TS in FD can get a better performance with the same channel state. It should be noted that the maximum value is obtained by the channel estimation method with a higher channel taps when the TS length hold fixed.

Figure 7 depicts the BER versus the SNR by using optimal TS in FD with the same number of channel taps but different number of TS symbols. The number of channel taps was $J=3$, but the TS lengths are $N=6,8,10$, respectively. It can be seen from Figure 7 that the BER decreases with the increase of the SNR. It should be noted that, the optimal TS with the less number of symbols can obtain the better BER performance under circumstances of the number of channel taps held fixed.

\section{Conclusions}

This article investigates the TS-based channel estimation in FD for indoor WOC systems. Firstly, the mathematical model is established for indoor WOC systems. Based on the established link model, three channel estimation methods, such as the LS method, the MMSE method and the ORL-MMSE method are proposed. Moreover, this article evaluates the performance of the proposed three methods by computer simulation, measuring in terms of MSE and BER, respectively. The mathematical analysis and the simulation results show that the LS estimator performs the worst of all methods and it has a lowest computational complexity, however. The MMSE estimator yields the best performance with its relatively higher complexity. The OLR-MMSE estimator gives the best trade off between performance and complexity. Based on the CRB theorem, a performance measure has been proposed to assess the quality of pilot symbols for FD channel estimation. Then, optimal TSs are found by a computer search. It has been demonstrated that the proposed method for searching the optimal TSs based on FD techniques invites a lower complexity search strategy compared with TD techniques. It should be noted that the optimal TSs in FD have a better BER performance compared to the TSs in TD. The minimum value is obtained by the channel estimation method with a lower channel taps when the TS length hold fixed. Moreover, with the number of channel taps held fixed, the longer the TS length is, the better the channel estimation performance can be obtained.

\section{Competing interests}

The authors declare that they have no competing interests. 


\section{Acknowledgements}

This work is supported by National Nature Science Foundation of China (No. 61102068, No.61172077 and No. 60972023), China Postdoctoral Science Foundation (No. 20110490389), Research Fund of National Mobile Communications Research Laboratory, Southeast University (No. 2012A06), the Research Fund for the Doctoral Program of Higher Education (No. 20113218120017), the open research fund of the State Key Laboratory of Integrated Services Networks, Xidian University (ISN12-11), NUAA Research Funding (NS2011013) and the Fundation of Graduate Innovation Center in NUAA (No.kfjj20110213 and No.kfjj20110129).

\section{Author details}

${ }^{1}$ College of Electronic and Information Engineering, Nanjing University of Aeronautics and Astronautics, Nanjing 210016, China. ${ }^{2}$ State Key Laboratory of Integrated Services Networks, Xidian University, Xi'an 710071, China. ${ }^{3}$ National Mobile Communications Research Laboratory, Southeast University, Nanjing 210096, China.

Received: 2 September 2011 Accepted: 23 September 2012 Published: 30 October 2012

\section{References}

1. JM Kahn, JR Barry, Wireless infrared communications. Proc. IEEE. 85(2), pp. 265-298 (1997)

2. K Langer, J Grubor, Recent developments in optical wireless communications using infrared and visible light, in International Conference on Transparent Optical Networks, vol. 3, Rome, July 2007) pp. 146-151

3. KNishida, S Miyamoto, K Tsukamoto, N Morinaga, A proposal of multi-beam transmitter for non-directed diffuse indoor optical wireless communication system, in Seventh IEEE International Symposium on Personal, Indoor and Mobile Radio Communications, vol. 1, Taipei, Oct 1996) pp. 242-246

4. FJ Lopez-Hernandez, CF Pozuelo, AS Galdon, Diffuse optical wireless communications for domotic indoor networks, in IEEE International Symposium on Consumer Electronic, Reading UK, Sept 2004) pp. 169-173

5. JB Carruthers, P Kannan, Iterative sitebased modeling for wireless infrared channels. IEEE Trans. Antenn. Propag. 50(5), pp. 759-765 (2002)

6. JR Barry, JM Kahn, EA Lee, DG Messerschmitt, High-speed nondirective optical communication for wireless networks. IEEE Netw. Mag. 5(6), pp. 44-54 (1991)

7. P Hoeher, S Kaiser, P Robertson, Pilot-symbol-aided channel estimation in time and frequency, in IEEE GLOBECOM, New York, 1997) pp. 90-96

8. W Chen, U Mitra, Training sequence optimization: comparisons and an alternative criterion. IEEE Trans. Commun. 48(12), pp. 1987-1991 (2000)

9. H Arslan, GE Bottomley, Channel estimation in narrowband wireless communication systems. Wirel. Commun. Mobile Comput. 1(2), pp. 201-219 (2001)

10. SN Crozier, DD Falconer, SA Mahmoud, Least sum of squared errors (LSSE) channel estimation. IEE Proceedings F Radar and Signal Processing. 138(4), pp. 371-378 (1991)

11. JB Wang, Y Jiao, XY Dang, M Chen, XX Xie, LL Cao, Training sequence based channel estimation for indoor visible light communication system. Optoelectron. Lett. 7(3), pp. 213-216 (2011)

12. JB Wang, Y Jiao, M Chen, Optimal training sequences for wireless optical communications. J. Opt. A: pure and applted optics. 14(1), p. 015401 (2011)

13. JB Wang, Y Jiao, XY Song, XB Zhao, M Gu, M Chen, XX Xie, M Sheng, Complementary-sequences-based channel estimation for diffuse wireless optical communications. Opt. Eng. 50(7), pp. (075003)1-6 (2011)

14. C Tellambura, MG Parker, YJ Guo, Optimal sequences for channel estimation using discrete fourier transform techniques. IEEE Trans. Commun. 47(2), pp. 230-238 (1999)

15. Li Ye, Pilot-symbol-aided channel estimation for OFDM in wireless systems. IEEE Trans. Vehicular Tech. 49(4), pp. 1207-1215 (2000)

16. JD Zhang, BY Zheng, Overview of pilot-aided channel estimation in OFDM. J. China Inst. Commun. 24(11), pp. 116-124 (2003)

17. H-N Lee, GJ Pottie, Fast adaptive equalization/diversity combining for time-varying dispersive channels. IEEE Trans. Consum. Electron. 46(9), pp. 1146-1162 (1998)
18. SQ Wang, A Abdi, A periodic complementary sets of sequences-based MIMO frequency selective channel estimation. IEEE Commun. Lett. 9(10) pp. 891-893 (2005)

19. AV Aho, JE Hopcroft, JD Ullman, The design and analysis of computer algorithms. (Addison-Wesley, Reading, MA, 1974)

20. TH Cormen, CE Leiserson, RL Rivest, Introduction to Algorithms. (MIT Press, Cambridge, 1994)

21. SM Kay, Fundamentals of Statistical Signal Processing: Estimation Theory (Prentice-Hall, NJ, 1993)

doi:10.1186/1687-1499-2012-326

Cite this article as: Wang et al.: Training sequence based frequency-domain channel estimation for indoor diffuse wireless optical communications. EURASIP Journal on Wireless Communications and Networking 2012 2012:326.

\section{Submit your manuscript to a SpringerOpen ${ }^{\mathcal{O}}$ journal and benefit from:}

- Convenient online submission

- Rigorous peer review

- Immediate publication on acceptance

- Open access: articles freely available online

- High visibility within the field

- Retaining the copyright to your article

Submit your next manuscript at $\gg$ springeropen.com 